\title{
La polisemia y la lingüística de gentrificación*
}

The polysemy and linguistics of gentrificación

Rafael Andrés Barrera Gutiérrez ${ }^{\star *}$

\section{Resumen}

El estudio en Colombia del fenómeno de la gentrificación es reducido y por lo mismo se han utilizado diferentes términos para designarlo. El presente artículo hace una revisión sobre el origen del término gentrification e intenta explicar su significado y el contexto en que se desarrolló. Tiene como objetivo revisar la pertinencia de usar traducciones en castellano que apuntan a darle un significado particular al fenómeno. Se sostiene que el término de "gentrificación", que se viene utilizando por parte de algunos estudiosos del tema, es el que mejor se acerca a expresar el fenómeno que se pretende estudiar. Además de una connotación ideológica, el término conceptualiza un proceso que no es homogéneo entre ciudades ni dentro de las mismas, donde confluyen intereses tanto públicos como privados, que no solo trata del cambio del territorio sino de las percepciones y los comportamientos que surgen alrededor de este y que en general resulta excluyente, diferenciador y enfocado en el consumo.

Palabras clave: ciudad; gentrificación; gentry; vivienda; conflicto de clases.

\begin{abstract}
The phenomenon of gentrification has received little attention in Colombia; therefore, different terms have been used to designate it. This paper reviews the origin of the term gentrification and tries to explain its meaning and the context in which it was developed. Also, the paper analyzes the relevance of its use in translations in Spanish that aim to give a particular meaning to the phenomenon. It is argued that the term "gentrificación", which has been used by some scholars, is the best term in Spanish to express the phenomenon to be studied. In addition to an ideological connotation, the term conceptualizes a process that is not homogeneous, neither among cities nor inside them, where public and private interests are present. It approaches not only a change in territory, but also a change in perception and behavior that emerges around the theme and that is usually exclusionary, differentiating and consumption-centered.
\end{abstract}

Keywords: city; gentrification; gentry; housing; class conflict. 
"¿Es la gentrificación una palabrota?" Así se titula el segundo capítulo del libro de Neil Smith, La nueva frontera urbana (Smith, 2012), donde da a entender que el vocablo mismo es importante debido al significado que tiene en relación a lo que implica el fenómeno en términos de su escala, del reconocimiento y entendimiento del mismo por parte de aquellos que se ven involucrados o no en este.

El estudio del fenómeno de la gentrificación en las metrópolis de Colombia es reciente y reducido. Como tal se ha relacionado con diferentes vocablos que indican por un lado lo innegable del fenómeno en sí, el reconocimiento y la comprensión del fenómeno mismo por parte de los estudiosos del tema y refleja en general una visión negativa del fenómeno.

Gentrification es un término que induce a pensar en la ciudad, en lo que comprende su territorio, sus fronteras, sus cambios físicos, sus progresos y retrocesos, su gente, sus conflictos, sus dinámicas sociales y económicas cambiantes, el efecto que en ella tiene el paso del tiempo y de los hombres con sus ideas y temores, en su cotidianidad, en un lenguaje, en un estilo de vida y en alternativas de vida.

El concepto de gentrification permite acercarse con precisión al análisis y entendimiento de la realidad que envuelve la regeneración de la ciudad o de alguna de sus zonas y su (re)descubrimiento por una parte de sus habitantes. Pero también es considerada una palabra "vedada", "sucia" o "fea" para promotores inmobiliarios, políticos y financieros, dado que conceptualiza un proceso con múltiples facetas dentro del desarrollo capitalista, que en particular se encuentra rodeado de conflicto social y que llama la atención sobre la importancia que tienen las zonas centrales de una ciudad (Lima, 2008; Smith, 2002, citado por Slater 2011; Smith, 2012).

Según Lima (2008) "el concepto de gentrification tiene una connotación 'ideológica', [cuyo] análisis seminal lo da Federico Engels". En la Contribución al problema de la vivienda de Engels este sostuvo:

Entiendo por Haussmann ${ }^{1}$ la práctica generalizada de abrir brechas en barrios obreros, particularmente los situados en el centro de nuestras grandes ciudades, ya responda esto a una atención de salud pública o de embellecimiento 0 bien a una demanda de grandes locales de negocios en el centro, o bien a unas necesidades de comunicaciones, como ferrocarriles, calles, etc. El resultado es en todas partes el mismo, cualquiera que sea el motivo invocado: las callejuelas y los callejones sin salida más escandalosos desaparecen y la burguesía se glorifica con un resultado tan grandioso; pero.... callejuelas y callejones sin salida reaparecen prontamente en otra parte, y muy a menudo en lugares muy próximos. (1980 [1873], p. 205)

Es ampliamente reconocido que la acuñación del término gentrification se atribuye a la socióloga alemana Ruth Glass (1964) quien de acuerdo con Slater (2011, p. 571), identifica el proceso de gentrification como "un vínculo entre lucha de clases y vivienda [...], en donde se ponen de manifiesto las inequidades e injusticias sociales creadas por las políticas y los mercados de tierra urbana" que recaen en las personas de más bajos ingresos y redundan en el deterioro de sus condiciones materiales de vida. Este empeoramiento material se lleva a cabo en algunas ocasiones de manera 
repentina y en todas las circunstancias incide de manera directa en el rompimiento de los lazos que construyen el tipo de tejido social que determina a las pequeñas comunidades y las familias.

Smith (2012, cap. 1) deja ver que la gentrification implica un proceso de conquista, de desarraigo, de uso de la fuerza, que no implica todas las veces el empleo directo de la violencia pero si el empuje directo hacia la pobreza, con ayuda de una racionalidad perversa, y el desplazamiento de la población gracias al uso de mecanismos indirectos permitidos por el mercado y el Estado. Esto acarrea un incremento en el costo de vida para algunos pobladores que no ven otro remedio que irse. Lo anterior permite concluir que la gentrificación es parte de la construcción de un nuevo orden, que diferente a lo que se pudiera creer resulta menos civilizado, menos incluyente y más abierto al abuso del capital (Smith, 2012, p. 72), básicamente porque detrás de la mayoría de las acciones realizadas opera la lógica de la ganancia pecuniaria que se esconde detrás de un discurso incluyente de mejoramiento del uso del espacio.

En la acepción original se evidencia el carácter conflictivo que subyace tras la apropiación del territorio, y que toma la forma de conflicto de clases en el caso de la apropiación del suelo urbano. Sin embargo, el uso de términos que pretenden acercarse al fenómeno sin estudiar realmente su significado y el contexto particular en que se desarrolla, aleja al lector del entendimiento real del fenómeno y cubre con un velo las implicaciones que el fenómeno tiene a nivel socio-espacial, económico, cultural y político.

\section{Términos usados en castellano para designar el proceso de gentrification y el caso de Colombia}

Para acercarse al estudio del fenómeno de gentrification el término se ha traducido como gentrificación, aburguesamiento, yupificación, elitización o aristocratización. Las expresiones se han utilizado indistintamente y, en general, no se encuentra correspondencia entre el fenómeno y el significado particular que cada palabra tiene. En buena parte de los casos, dichos vocablos solamente designan un aspecto del fenómeno aunque se intente dar una explicación más amplia.

En gran parte de la literatura se puede observar que gentrificación alude a conflicto y segregación, en particular porque el proceso mismo implica una reconstrucción del espacio y de las relaciones que se dan en torno al mismo. En ese sentido González G. (2011) utiliza el término gentrificación y lo asocia (para el caso colombiano) con los problemas de exclusión y expulsión que el fenómeno manifiesta. Contreras (2010), al realizar un análisis para el barrio El Cartucho de Bogotá, ubicado en el centro de la ciudad, del papel que tiene la intervención pública en la disposición del espacio y la revalorización del territorio utiliza la palabra gentrificación como un proceso asociado con el fenómeno conocido en Colombia como "limpieza social", en el que es evidente el papel jugado por el Distrito y los privados alrededor del proyecto de recuperación del espacio público y el proceso de expulsión de los habitantes del sector. 
En las operaciones de renovación urbana intervienen el Estado y los privados (Cuenya, 2011; Badyina y Golubchikov, 2005) que inciden en la generación de lo que Neil Smith denominó una "brecha de renta" (rent gap), y que el conflicto surge cuando hay desplazamiento o riesgo de ver minados los intereses de sectores amenazados por la gentrificación. "Es la existencia de la diferencia potencial de renta en el centro lo que facilita la traducción de los procesos sociales, así como también económicos, más graduales, al desplazamiento espacial de algunas actividades residenciales, recreativas y laborales" (Smith, 2011, p. 187). Para el caso, Mendes (2011) considera que "las intervenciones públicas desencadenan procesos contradictorios de expulsión y reapropiación, en donde es evidente una lógica de mercado a favor de los consumidores que va en contra de las clases menos favorecidas" (Mendes, 2011, p. 481), y que según Smith (op cit, p. 123) se pueden efectuar sin la ayuda de los agentes inmobiliarios.

De Urbina (2012) da cuenta de los diferentes términos usados y aunque no se detiene a examinar su pertinencia y adopta en su escrito el término gentrificación, de manera detallada da testimonio sobre la manera en que el fenómeno se manifiesta en el centro histórico de Bogotá a partir de un análisis exhaustivo del estado físico del parque inmobiliario. Sus resultados dan cuenta de la trascendencia social de un fenómeno que es necesario comprender a profundidad como parte de un proceso reciente en la región metropolitana de Bogotá. Lulle (2008) resalta que en Bogotá "existe un desfase importante entre las necesidades y aspiraciones de las personas que viven en el centro y el proyecto de los planificadores de cambiar la población" (Lulle, 2008, p. 67). Se refiere al fenómeno como "elitización o gentrificación" y muestra que los procesos de cambio en el centro de Bogotá en donde se conjugan el accionar público y privado, de nacionales y extranjeros, van de la mano con sus dinámicas sociales y culturales resultan diversas y requieren de un cuidadoso estudio para su comprensión. Se reconoce en estos trabajos que el particular deterioro de construcciones que están en manos tanto de particulares como de entidades oficiales por un lado, y procesos de intervención concluidos y en marcha, por otro, son muestra de que el fenómeno no es homogéneo en la zona. De hecho, las reglas para la conservación del patrimonio arquitectónico han redundado, en no pocos casos, en el deterioro del mismo debido al mal diseño de los estímulos y las regulaciones.

La gentrificación es un "fenómeno [...] enraizado inherentemente en la clase pero [...] es muchas otras cosas [...] Las clases deberían ser vistas no como casilleros, o series sobredeterminadas con límites precisos y reglas binarias exactas de inclusión y exclusión. Las clases se asemejan, más bien, a conjuntos difusos que se definen más o menos claramente dependiendo de las condiciones sociales, económicas, políticas e ideológicas. (Smith, 2012, pp. 178-179)

Las clases no son iguales ni entre países, ni entre ciudades, ni entre sectores de la ciudad por ende el proceso de gentrificación tampoco puede ser igual en las mismas. La actividad dominantes de la clase trabajadora varía entre vecindarios, los estilos de vida y los costos asociados a estas cambian entre ciudades, 
lo cual hace que el fenómeno mismo de gentrificación y el arreglo posterior que surge sea también distinto (Cole, 1985).

Por su parte, González C. (2008) iguala el término gentrificación a aburguesamiento y muestra que si bien es un término que ha adquirido importancia siguen presentándose esfuerzos por ampliar su significado y en particular por restarle la negatividad con el que se le asocia, del cual este trabajo forma parte puesto que sostiene que el fenómeno es "una apuesta por la diversidad social y estética variada" (González C., 2008, p. 138). "La gentrificación no puede traducirse a la mera relocalización del capital a las aceras centrales de las ciudades, también abarca simultáneamente la formación de grupos y la diferenciación de ellos mismos de otros" (Clerval, p. 170, citada por Giraud, 2013).

Nates (2008) considera el término "elitización o gentrificación" y utiliza los adjetivos de "normal y marginal" para diferenciar a las personas por nivel de ingreso y por el tipo de actividad que registran (en particular artistas e intelectuales) para el segundo (Nates, 2008, p. 256). Considera que la gentrificación puede darse desde la yupificación sin dar mayor explicación a su significado. Estudia el fenómeno denotado como gentrificación rural (rural gentrification) para denotar que el fenómeno no es exclusivo de las ciudades y que el término elitización "produce un carácter social excluyente [...] donde los lugares elegidos son convertidos en objeto estéticamente relevante para los mismos" (Nates, 2008, p. 266) y que permite expresar por parte de sus poseedores la distinción de clase.
Smith (2012, p. 159), en este último sentido, aclara lo que el término yupi trae consigo. Al momento de acuñarse "en 1983 para referirse a aquellos jóvenes profesionales con movilidad ascendente pertenecientes a la generación nacida después de la Segunda Guerra Mundial, ya había adquirido una amplia difusión". Se asocia con una nueva clase media ascendente que se distingue por su edad, su movilidad social, un modo de vida urbano y el consumo "empedernido", que bien podría asociarse con el consumo ostentoso del que hablaba Thorstein Veblen en su Teoría de la clase ociosa.

Se asocia la gentrificación con el poder del consumo y con la capacidad transformadora de este. No solo se cambia el aspecto físico del territorio, se cambian las relaciones entre los habitantes alrededor de los mismos, no solo por el cambio en el espacios y porque los habitantes sean diferentes, sino por "la percepción de lo que cada persona tiene sobre lo que está ocurriendo en el sector" (Cole, 1985, p. 153) aun cuando los habitantes puedan llegar a ser los mismos.

Al dejar por fuera del análisis las luchas que surgen por la apropiación del espacio, su control y su uso en un contexto capitalista, se da margen para utilizar conceptos como conservación urbana, mejoramiento urbano, reconquista urbana, regeneración urbana, remodelación urbana, rescate urbano y salvamento urbano (Lima, 2008). También se podrían agregar en este sentido términos como revitalización, renovación, residencialización y renacimiento urbanos como términos usados en esta misma vía, los cuales recogen el carácter amable y benéfico, tanto para la 
ciudad como para los habitantes que rodean dichos lugares, que tienen los procesos de intervención. En este sentido, Gagliardi (2001, p. 123) "analiza las estrategias de intervención en áreas históricas centrales y propone relativizar el carácter exclusivamente mercantil que tiende a envolver las prácticas de renovación urbanas contemporáneas". Para esto utiliza el caso de renovación del centro histórico de la ciudad italiana de Génova como ejemplo exitoso de iniciativa comunitaria. Dicho proyecto financiado con recursos del Fondo Estructural de la Unión Europea tenía como "propósito [...] el desarrollo de las regiones menos desarrolladas, una reconversión económica y social de zonas con problemas estructurales y la creación de puestos de trabajo" (ibid, p. 131). El alcance del proyecto se da básicamente porque el sentido del mismo giraba alrededor de la cultura y el patrimonio, la cual se convirtió en elemento articulador de clases e intereses y ante todo por el origen mismo de los fondos lo cual le quitó el carácter de negocio.

Este último caso va en contravía a lo expuesto por Carman (2011) en donde "Io patrimonial y lo cultural resultan argumentos en apariencia neutrales para echar intrusos, pues se los desaloja por su propio bien, o para defender el espacio público", pero en realidad es usado "como un recurso político para la expulsión (vía mercado o, según el autor, desalojo light) de los habitantes innobles $y$, paralelamente, los ocupantes también realizan determinados usos e interpretaciones del patrimonio local - en algunos casos como recurso de distinción - para evitar ser desalojados y permanecer en el barrio".

\section{Un acercamiento a la relación entre gentrification y gentry y el vocablo más apropiado en lengua castellana}

El concepto de gentrification "no cuenta con una delimitación especial concreta" (Manrique, 2013, p. 212) y su estudio suscita interrogantes diversos tales como las características particulares que el fenómeno tiene en los distintos lugares, los costos que implica en la actualidad y en el futuro, además de la etapa del proceso que cada lugar está experimentando, así como su velocidad y el papel que juegan el sector público y el privado en la misma y en la totalidad del proceso. Ahora bien, en general son escasos los trabajos que se pueden encontrar sobre lo adecuado de encontrar un término en castellano que designe de manera precisa el fenómeno, en vista de la dificultad que presenta el traducir un término que tiene en su lengua original un significado particular y en donde, como sostiene Duque (2010), su relevancia no reposa en el debate alrededor de una buena traducción del vocablo gentrification, sino a la conveniencia de realizar dicho esfuerzo sin un trabajo de reflexión previo.

En este mismo sentido, Duque realiza un análisis sobre lo que ha pasado alrededor del fenómeno y del término. Muestra el intento de traducción al castellano por Luz Marina García quien apuesta por elitización como el término más adecuado puesto que la idea o noción de élite apunta a resaltar la importancia de un grupo particular, con capacidad de convicción e influencia en la toma de decisiones (García, 
2001). Pero, en general, no argumenta de manera contundente con respecto a la pertinencia de la utilización del término y su posibilidad de explicación general del fenómeno. Duque (2001) después de argumentar por qué no resultan pertinentes términos como aristocratización, hidalguización, enhidalgamiento, elitización y aburguesamiento, considera que el idioma castellano es menos maleable cuando se trata de crear nuevas palabras y expresiones. De igual manera considera que esta palabra, en particular, designa un proceso básicamente que pertenece a un contexto extranjero que lo define, y que previo a la creación de un nuevo vocablo es preciso darle un sentido propio al término por lo que resulta oportuno estudiar de manera más concienzuda el fenómeno. Para Hamnett (2003):

[...] el uso del término gentrification por parte de Ruth Glass, el cual algunas veces causa molestia o desconcierto a los académicos, quienes prefieren el término de revitalización o renovación, fue deliberadamente irónico e ingeniosamente divertido. Enraizado en las complejidades de la clase rural tradicional inglesa, el término fue designado para señalar la emergencia de una nueva "gentry urbana", paralela a la "gentry rural" de los siglos XVIII y XIX de los lectores de Jane Austen, quienes comprendían la clase por debajo de la aristocracia terrateniente, pero sobre terratenientes agricultores y campesinos. (Hamnett, 2003, p. 2401)

En este sentido, Sargatal (2000) anota que Glass usó esta expresión al comparar el hábito de la gentry de las áreas rurales de Inglaterra, que conformaban la clase media inglesa, de tener una vivienda tanto en la ciudad como en el campo, hecho que se observó en Londres en los años 60.

Al realizar una exploración breve sobre el origen de la gentry se encuentra que es una referencia a una formación social que se desarrolla en Inglaterra en el periodo final de la baja Edad Media y que resultó de cambios políticos, sociales y económicos a finales del siglo XVI. Para Coss, el término gentry,

[...] en su sentido amplio es un término que brinda más vaguedad que ayuda y en particular para el periodo de la Edad Media, aun cuando como símbolo del pasado es eminentemente significativo para sus audiencias. Pero su uso en la actualidad se presenta porque el lenguaje común permite su uso como un término social vivo. Este término vino a ser usado, para cubrir al menor estrato de los terratenientes de la sociedad una vez la hidalguía (Nobility) llegó a restringirse a la nobleza, y en este sentido su uso continúa hasta el presente. Sin embargo esto ha ocurrido lentamente. A finales del siglo XVI y comienzos del XVII los comentaristas preferían escribir de la hidalguía (Peerage) como la nobleza mayor y de los caballeros, títulos y gentlemen (para ser unido después de 1611 por los títulos) como el título menor. En el lenguaje común, sin embargo, gentry, una vez sinónimo de nobleza, vino a significar la menor nobleza. Una vez intercambiados, estos términos se vuelven complementarios: nobleza y gentry. $(1995$, p. 39) 


\section{Sobre la gentryy su relación con el territorio y las construcciones}

Coss (1995, p. 40) sostiene que "los historiadores han confinado sin significado su uso del término gentry a Inglaterrra o a la época cuando este era un término social vivo. Pero al contrario de esto, el término ha sido prontamente exportado y ha sido usado transhistóricamente y transculturalmente en el supuesto de que sería fácilmente entendido". Al preguntarse si esto no es una mera ilusión, en cuanto respecta a la alta Edad Media, se responde que este no era de hecho un término social vivo, al menos no en los términos actuales. "La palabra gentry se remonta a gentrice y su uso común era para indicar "bien nacido" (gentle birth) y un alto rango o para describir las cualidades compartidas por la gente" (1995, p. 40). Por otro lado, al cuestionarse quién conforma la gentry considera que un acercamiento básico es igualar gentry a "gentileza, gracia, donaire, garbo (gentility); la gentry no son todos aquellos que son aceptados como tales, o quienes pretenden serlo" (1995, p. 41).

Ahora bien, para este autor las características que permanecen de la gentry pueden encapsularse en una sola palabra: territorialidad. Esta resulta crucial para el entendimiento de la gentry como una construcción social, y lo que lo distingue dentro de la territorialidad es su carácter colectivo. Para Tawney (1941), la gentry inglesa conformada por individuos que, aunque variaban en riqueza y entre quienes había ambigüedades, tenían un centro sólido que consistía en la propiedad de tierras sobre la caballería (que coincide hasta cierto punto con la territorialidad de Coss). El avance de esta clase se debe en parte a la agricultura como un negocio que estaba acompañado por un segundo movimiento que reflejó su influencia y consolidó sus resultados. "Este movimiento fue la creciente rapidez con que la tierra estaba cambiando de manos. La tierra se transa en forma de capital, y en muchas sociedades, así toma su forma más importante. Pero no es sólo un bien sujeto de transacción, es un instrumento de prestigio social y poder político" (Tawney, 1941, p. 19), cuya influencia se extendía a la educación, las profesiones, las artes, y en particular a la arquitectura.

Sobre este último punto, Mercer (1954, p. 11) hace una diferenciación entre aristocracy y gentry y basado en el análisis del desarrollo arquitectónico de las construcciones en Inglaterra en los siglos XVI y XVII intenta mostrar el tipo de desarrollo social que se refleja en las mismas. De manera particular, al sustentar su análisis en el estudio de los planos de las casas invita a una reflexión sobre las cambiantes relaciones de sus constructores, y procura mejorar de esta manera la comprensión de las relaciones que al apoyarse solamente en fuentes documentales no resultan libres de ambigüedades. Por gentry quiere decir todos aquellos que no eran dependientes de, o recibían favores de la Corona, mientras que por courtiers (cortesanos) el autor quiere decir todos aquellos que estaban atados políticamente a la Corona, o que eran dependientes de sus oficinas o garantía y favores, o que estaban extrayendo sus riquezas del sistema de restricciones impuesto por esta. 
Para Mercer (1954, p. 12) "estos últimos necesitaban que sus casas fueran amplias, para acomodar a sus numerosos sirvientes y seguidores, y de esta manera expresar su posición en el mundo, a la vez que para agasajar, si fuera necesario, a sus soberanos de quienes recibían los favores". Luego surgen clases que hacen su fortuna sin depender de los favores de la Corte, sino explotando el desarrollo económico dentro del país. Esta fue la gentry del país, "muchos de los hacendados, el conjunto de comerciantes y manufactureros, que se distinguían de las clases superiores de grandes comerciantes (Merchant-princes), financieros e industriales con patentes - y una gran sección de abogados practicantes" (1954, p. 20). Se caracterizaron por ser un grupo heterogéneo a diferencia de los cortesanos y sus construcciones por ende, diversas y con variaciones "debido precisamente a la atención que mucho de ellos prestaban a los costos de la construcción" (1954, p. 20). Los miembros de la gentry "construían para ellos, no para entretener a las cortes y tampoco resultaban, como los pequeños terratenientes, meros constructores de casas grandes y confortables para labradores" (p. 21).

\section{A manera de conclusión}

El término gentrification no hace referencia al proceso al que alude Nates (2008) de "elitización de territorios que han sido habitados en lugares urbanos, rurales y rururbanos por poblaciones con poder adquisitivo bajo o medio, y que han sido adquiridos por poblaciones de sectores medios altos que según los casos presentan características sociales y culturales" (2008, p. 255), puesto que desde la concepción original esta elitización implicaría que son los de la élite quienes finalmente llegarían a estos lugares, y como indica el término, haría referencia a una minoría selecta con un estatus superior que se destaca por su sabiduría y virtud (cursivas mías). Se puede considerar como característica básica de la gentry que sus miembros poseen una capacidad económica mínima que determina su diferenciación del resto. No es el tipo de actividad a la que se dedican, ni el tipo de costumbres (aunque realmente se esperaría que fuera así) que tienen los miembros de la gentry lo que indica su rango y clase, sino el patrón de consumo que presentan lo que en buena medida apunta a diferenciarlos.

La gentrificación supone una nueva diferenciación del paisaje cultural, social y económico en donde los espacios creados sugieren una nueva forma de acumulación y de consumo que señalan una particular relación de poderes y luchas por el espacio, donde el proceso mismo y sus resultados pueden ser vistos como una "historia elegante de renovación para algunos, y una desgraciada pieza de tiranía para otros" (Badyina y Golubchikov, 2005, p. 114).

La conveniencia de ajustar un término en castellano que designe el significado que la palabra tiene en su lengua original, no resulta fácil ni conveniente, y posiblemente mutatis mutandis sea conveniente adoptar definitivamente el término gentrificación como se viene haciendo por parte de algunos estudiosos del tema, haciendo la salvedad que este vocablo a pesar de sonar parecido al original tiene una connotación distinta y que no designa todavía con propiedad 
el fenómeno que se pretende estudiar. El proceso, para el caso colombiano no termina de definirse de manera precisa y el fenómeno no solo abarca aspectos económicos sino sociales, culturales y políticos que vale la pena considerar para entender el carácter particular que el mismo tiene en cada lugar en que se presenta.

\section{Rafael Andrés Barrera Gutiérrez}

Universidad Externado de Colombia, Facultad de Economía. Bogotá, Colombia.

rafael.barrera@uexternado.edu.co

\section{Notas}

(*) Una versión inicial de este artículo se presentó con el título de "Gentrification: sobre el origen del término y el vocablo más adecuado en lengua castellana" para su publicación en la revista Papeles de Coyuntura de la Universidad Piloto de Colombia, en noviembre de 2013.

$(* *)$ El autor agradece a Oscar A. Alfonso por todo el apoyo, sugerencias y ayuda brindados, y al comité editorial por los comentarios e indicaciones.

(1) Aquí se hace alusión a Georges-Eugène Barón Haussmann quien fuera un funcionario público francés encargado por Napoleón III de realizar hacia 1852 un proceso de modernización de París. Este consistió, en parte, en eliminar calles antiguas y casas de apartamentos que fueron reemplazados por un nuevo trazado de vías (particularmente anchas y largas avenidas), que iban de la mano con el desplazamiento de obreros del centro y el impedimento de ubicación de barricadas que favorecieran levantamientos populares.

\section{Referencias}

BADYINA, A. y GOLUBCHIKOV, O. (2005). Gentrification in Central Moscow: a market process or a deliberate policy? money, power and people in housing regeneration in Ostozhenka. Geografiska Annaler. Series B, Human Geography, v. 87, n. 2, Special Issue: Power Over Time-Space: The Inaugural Nordic Geographers Meeting, pp. 113-129.

CARMAN, M. (2011). El proceso de ennoblecimiento y la salida negociada de los innobles en Buenos Aires. Cadernos Metrópole, São Paulo, v. 13, n. 25, pp. 257-278.

COLE, D. (1985). Gentrification, social character, and personal identity. Geographical Review, v. 75, n. 2, pp. $142-155$. 
CONTRERAS S, C. (2010). Estética del residuo: el proceso de gentrificación de El Cartucho en Bogotá. Blog disponible en: http://pervisiones.blogspot.com/2009/10/estetica-del-residuo-el-procesode.html. Acceso en: 25 ago 2013.

COSS, P. R. (1995). The formation of the english gentry. Past \& Present, n. 147, pp. 38-64. Mayo, Oxford University Press.

CUENYA, B. (2011). Grandes proyectos e impactos en la centralidad urbana. Cadernos Metrópole. São Paulo, v. 13, n. 25, pp. 185-212.

DE URBINA, A. (2012). El Centro histórico de Bogotá “de puertas para adentro": ¿el deterioro del patrimonio al servicio de la gentrificación? Cuadernos de vivienda y urbanismo, v. 5, n. 9, pp. 46-69.

DUQUE, R. (2010). La difusión del concepto gentrification en España: reflexión teórica y debate terminológico. Revista bibliográfica de Geografía y Ciencias Sociales, v. XV, n. 875. En: Serie documental de Geo Crítica. Cuadernos Críticos de Geografía Human. Universidad de Barcelona.

ENGELS, F. (1873/1980). Contribución al problema de la vivienda. Obras Escogidas (en tres tomos) de C. Marx y F. Engels. Tomo II. Edición Progreso, Moscú. Disponible en: http://www.marxists.org/ espanol/m-e/oe/pdf/oe3-v2.pdf Acceso en: 20 sept 2013.

GAGLIARDI, C. (2011). Um grande projeto entre o mar e as colinas: a renovação urbana da cidade italiana de Gênova. Cadernos Metrópole, São Paulo, v. 13, n. 25, pp. 123-143.

GARCIA H., L. M. (2001). Elitización: propuesta en español para el término gentrification. Revista Bibliográfica de Geografía y Ciencias Sociales, Universidad de Barcelona, v. 6, n. 332. Disponible en: http://www.ub.edu/geocrit/bw-ig.htm. Acceso en: 25 ago 2013.

GIRAUD, C. (2014). Gentrification in Paris: the elite versus the people? Metropolitics, 29 January. Disponible en: http://www.metropolitiques.eu/Gentrification-in-Paris-theelite.html. Acceso en: 4 mayo 2014.

GLASS, R. (1964). "Introduction: Aspects of Change". London Aspects of Change: Centre for Urban Studies. London, Mac-Gibbon and Kee.

GONZÁLEZ C., M. M. (2008). Gentrification. Revista La Tadeo, n. 73, pp. 137-143. Universidad Jorge Tadeo Lozano.

GONZÁLEZ G., C. A. (2011). Gentrificación y otros exorcismos urbanos. Revista Dinero. Opinión on Line. Disponible en: http://www.dinero.com/opinion/opinion-on-line/articulo/gentrificacion-otrosexorcismos-urbanos/136981. Acceso en: 20 ago 2013.

HAMNETT, C. (2003). Gentrification and the Middle-class Remaking of Inner London, 1961-2001. Urban Studies, v. 40, n. 12 , pp. 2401-2426.

LEVY. F. J. (1982). How information Spread among the Gentry, 1550 -1640. The Journal of British Studies. Spring, University of Chicago Press, v. 21, n. 2, pp. 11-34.

LIMA, S. S. (2008). Y sin embargo,... se gentrifica. Análisis en torno al problema de la gentrification de la ciudad de México. Diez años de cambios en el Mundo, en la Geografía y en las Ciencias Sociales, 1999-2008. En: X COLOQUIO INTERNACIONAL DE GEOCRÍTICA. Actas. Universidad de Barcelona, 26-30 de mayo. Disponible en: http://www.ub.es/geocrit/-xcol/200.htm. Acceso en: 20 ene. 2013

LULLE, T. (2008). Prácticas y representaciones espaciales de los habitantes de Bogotá. Revista de la Organización Latinoamericana y del Caribe de Centros Históricos, n. 1, pp. 67-77. 
MANRIQUE, A. (2013). Gentrificación de la Candelaria. Reconfiguraciones del lugar de residencia y consumo de grupos de altos ingresos. Cuadernos de Geografía. Revista Colombiana de Geografía. Universidad Nacional de Colombia, Bogotá, v. 22, n. 2, pp. 211-234.

MENDES, L. (2011). Cidade pós-moderna, gentrificação e a produção social do espaço fragmentado. Cadernos Metrópole. São Paulo, v. 13, n. 26, pp. 473-495.

MERCER, A. E. (1954). Houses of the gentry. Past \& Present. Oxford Univeristy Press, n. 5, pp. 11-32.

NATES, B. (2008). Procesos de gentrificación en lugares suburbanos: presupuestos conceptuales para su estudio en Colombia. Artículo de investigación. Universidad de Caldas, Manizales, Grupo de Investigación Territorialidades.

SARGATAL B., M. A. (2000). El estudio de la gentrificación. Revista Bibliográfica de Geografía y Ciencias Sociales. Universidad de Barcelona, n. 228. Disponible en: http://www.ub.edu/geocrit/b3w-228. htm. Acceso en: 26 sept 2013.

SLATER, T. (2011). "Gentrification of the city". En: BRIDGE, G. e WATSON, S. (eds.). The New Blackwell Companion to the City. Blackwell Publishing. Blackwell Reference Online. Disponible en: http:// www.blackwellreference.com/public/book.html?id=g9781405189811_9781405189811. Acceso en: 20 ago 2013.

SMITH, N. (2012). La nueva frontera urbana. Ciudad revanchista y gentrificación. Madrid, Traficante de sueños.

TAWNEY, R. H. (1941). The Rise of the Gentry, 1558-1640. The Economic History Review. Blackwell Publishing, v. 11, n. 1, pp. 1-38.

Texto recebido em 12/out/2013

Texto aprovado em 17/mar/2014 